patients, with the ambition of developing a consensus document to support health care professionals in the future.

Methods This qualitative study involved four focus groups, and was conducted between March and October 2018. It involved a total of 27 expert participants IBD-specialist nurses $(n=8)$, gastroenterologists $(n=7)$, general practitioners $(n=5)$, colorectal surgeons $(n=2)$, pharmacists $(n=2)$, a histopathologist $(n=1)$ and clinical commissioning experts $(n=2)$. All participants were given pre-sessional information. Facilitated discussion was then undertaken between experts on key topic areas, and culminated in key themes, which were then further analysed.

Results The following themes were identified to influence patient care and outcomes: 1) Awareness. A lack of awareness particularly in primary care, and perceptions that MC is less common than conventional inflammatory bowel diseases were felt to be factors influencing diagnosis and management. 2) Symptom overlap with IBS. As symptomatology for both MC and IBS are similar, the expert group felt referrals to secondary care are likely to be restricted, leading to increased GP visits, enhanced use of over the counter medications, and diminished quality of life. 3) Faecal calprotectin (FC). The expert group felt referral practice from primary care was too reliant on FC as a discriminator, as active MC can have FC levels below the conventional referral thresholds. 4) Biopsies. The expert group identified that the diagnosis of MC may be missed as endoscopists neglect to take colonic biopsies, either through a lack of awareness, or determined by time and cost restrictions. 5) Treatment. This theme demonstrated significant variations in clinical practice, with an array of therapeutic interventions used to manage MC. Clinicians frequently lacked awareness of MC treatments and were not aware that budesonide is the only established treatment, as evidenced in randomised-controlled trials.

Conclusions This is the first study to examine service provision for MC in the UK. Key themes have been identified through expert opinion, demonstrating why optimal diagnosis and management of $\mathrm{MC}$ has been difficult to operationalise in the UK. These findings will be used to develop a consensus document that has benefits to healthcare professionals and patients.

\section{PTU-096 INTRODUCTION OF A GASTROENTEROLOGY OUTREACH SERVICE AND ITS IMPACT WITHIN A TERTIARY UNIVERSITY HOSPITAL}

Timothy Keen, Imdadur Rahman, Philip Boger, Nicholas Coleman, Bernard Stacey. University Hospital Southampton, NHS Foundation Trust, Southampton, UK

\subsection{6/gutjnl-2019-BSGAbstracts.455}

Introduction Hospital services are under increasing pressure which has led to an increased drive for earlier discharges. Various initiatives have begun to manage this. Within a UK tertiary hospital, a gastroenterology outreach team was formed from a pre-existing inpatient team alongside 2 remaining inpatient gastroenterology teams. This change coincided with the introduction of a new IT system with electronic triages and referrals. The aim of this service evaluation was to assess the impact on patient management, hospital stay and referring specialty satisfaction.

Methods The outreach team consisted of a consultant gastroenterologist and either a nurse specialist or junior doctor. The role of the outreach team was to review direct to gastroenterology triages within an acute medical admissions unit as well as all ward referrals. The direct to speciality triages were managed on the acute medical unit by the outreach team or admitted to a ward to be cared for by one of the inpatient teams. A retrospective analysis of a prospective kept database of all speciality triages and referrals was performed over a 15 month period from October 2017 to January 2019.

Results A mean increase of $15 \%$ per year in the number of direct to speciality triages and referrals was noted (mean 86/ month). $49 \%$ of referrals were from 3 specialities; cardiology $(25 \%)$, surgery (12\%) and respiratory (12\%). 100\% of referrals and speciality triages were seen within 24 hrs. Vomiting and/or dysphagia (33\%), diarrhoea (16\%), GI bleeding (14\%) and weight loss (12\%) account for the majority of speciality triages (mean 68/month). The outreach team managed 22\% of these without input from any inpatient teams. Following the introduction of the outreach team there has been a $14 \%$ reduction in the number of patients admitted to a ward from the acute medical unit (67\% to $53 \%)$, an $11 \%$ increase in patients discharged $<48$ hours ( $34 \%$ to $45 \%$ ) and a reduced length of hospital stay by 2 days (median of 4 to 2 days). A 1632 bed days saved per year equated to a cost saving of $£ 652800$ annually. Inpatient teams rated the outreach team as $9.2 / 10$ overall and at $8.7 / 10$ for patient management $(1=$ poor, $10=$ excellent).

Conclusions The outreach team has been successful in improving initial patient management which has subsequently reduced the number of speciality triages admitted to a ward, increased patient discharges $<48$ hours, reduced length of stay, saved bed days and increased satisfaction with the service. We believe if this model of patient care was introduced to other hospitals it will have similar benefits.

\section{PTU-097 LEICESTER CITY CCG EXPERIENCE: DO DIRECT PHONE CALLS TO NON-RESPONDERS IMPROVE BCSP UPTAKE?}

Hui Lin Lee*, Racheal Fenton, Shelley Mellor, Karen Emery-Downing, Richard Robinson. University Hospitals Leicester, Leicester, UK

\subsection{6/gutjnl-2019-BSGAbstracts.456}

Introduction Bowel Cancer Screening Programme (BCSP) has shown to be associated with a substantial stage shift in colorectal cancer diagnosis. In 2015/16, the overall percentage uptake for gFOBt bowel screening nationally was $56.4 \%$. There was a lower uptake across Leicester City CCG of $45.8 \%$. The aim of this project was to assess if direct phone calls to non-responders towards gFOBt bowel screening improve BCSP uptake locally.

Methods We identified individuals from 8 local GP surgeries who had not returned their BCSP gFOBt kit between September and October 2016. These individuals were all phoned by a nurse specialist. During the telephone call encounter, we discussed the importance of bowel cancer screening, checked if each subject had received the 1 st gFOBt kit, identified if there was any language barrier, offered further information (written/ picture booklets) and enquired if subjects would like another kit sent out again.

Results A total of 412 (202 females, 210 males) non-responders were identified. The mean and median age of the subjects was 60 years and 64 years respectively. A total of 580 phone 